\title{
MANIN'S CONJECTURE FOR A QUARTIC DEL PEZZO SURFACE WITH $A_{3}$ SINGULARITY AND FOUR LINES
}

\author{
by
}

Pierre Le Boudec

\begin{abstract}
We establish Manin's conjecture for a quartic del Pezzo surface split over $\mathbb{Q}$ and having a singularity of type $\mathbf{A}_{3}$ and containing exactly four lines. It is the first example of split singular quartic del Pezzo surface whose universal torsor is not a hypersurface for which Manin's conjecture is proved.
\end{abstract}

\section{Contents}

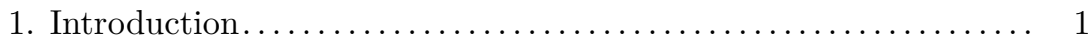

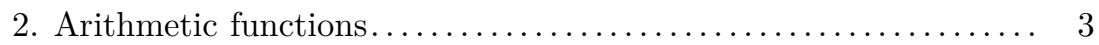

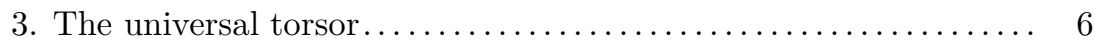

4. Calculation of Peyre's constant........................ 8

5. Proof of the main theorem........................... 8

References................. 16

\section{Introduction}

Manin's conjecture (see FMT89) gives a precise description of the distribution of rational points of bounded height on singular del Pezzo surfaces. More precisely, let $V \subset \mathbb{P}^{n}$ be such a surface defined over $\mathbb{Q}$ and anticanonically embedded and $U$ be the open subset formed by deleting the lines from $V$. We set

$$
N_{U, H}(B)=\#\{x \in U(\mathbb{Q}), H(x) \leq B\},
$$

where $H: \mathbb{P}^{n}(\mathbb{Q}) \rightarrow \mathbb{R}_{>0}$ is the exponential height defined by

$$
H\left(x_{0}: \ldots: x_{n}\right)=\max \left\{\left|x_{i}\right|, 0 \leq i \leq n\right\},
$$

for $\left(x_{0}, \ldots, x_{n}\right) \in \mathbb{Z}^{n+1}$ satisfying the condition $\operatorname{gcd}\left(x_{0}, \ldots, x_{n}\right)=1$. If $\widetilde{V}$ denotes the minimal desingularization of $V$ and $\rho=\rho_{\widetilde{V}}$ the rank of the Picard group of $\widetilde{V}$, then it is expected that

$$
N_{U, H}(B)=c_{V, H} B \log (B)^{\rho-1}(1+o(1)),
$$

where $c_{V, H}$ is a constant which is expected to follow Peyre's prediction Pey95.

2010 Mathematics Subject Classification. - 11D45, 14G05.

Key words and phrases. - Rational points, Manin's conjecture, del Pezzo surfaces, universal torsors. 
We are only interested here in singular del Pezzo surfaces of degree four. Their classification is rather classical and can be found in the work of Coray and Tsfasman CT88. Up to isomorphism over $\overline{\mathbb{Q}}$, there are fifteen types of such surfaces and they are categorized by their extended Dynkin diagrams which are the diagrams describing the intersection behaviour of the negative curves on the minimal desingularizations (see Der06b, Table 4]). Here is a quick overview of the available results concerning Manin's conjecture for singular quartic del Pezzo surfaces split over $\mathbb{Q}$. The conjecture is already known to hold for nine surfaces of different types. Using harmonic analysis techniques on adelic groups and studying the height Zeta function

$$
Z_{U, H}(s)=\sum_{x \in U(\mathbb{Q})} H(x)^{-s},
$$

Batyrev and Tschinkel have proved it for toric varieties BT98 (which covers the three types $4 \mathbf{A}_{1}, 2 \mathbf{A}_{1}+\mathbf{A}_{2}$ and $2 \mathbf{A}_{1}+\mathbf{A}_{3}$ ) and Chambert-Loir and Tschinkel have proved it for equivariant compactifications of vector groups CLT02 (which covers the type $\mathbf{D}_{5}$ ). Note that for a certain surface of type $\mathbf{D}_{5}$, la Bretèche and Browning have proved the conjecture independently BB07. Finally, the conjecture has been obtained for five other surfaces, a surface of type $\mathbf{D}_{4}$ by Derenthal and Tschinkel DT07, a surface of type $\mathbf{A}_{1}+\mathbf{A}_{3}$ by Derenthal [Der09, a surface of type $\mathbf{A}_{4}$ by Browning and Derenthal BD09 and two surfaces of respective types $3 \mathbf{A}_{1}$ and $\mathbf{A}_{1}+\mathbf{A}_{2}$ by the author [LB10]. These proofs are very different from those using the fact that the varieties considered are equivariant compactifications of algebraic groups. They all use a lift to universal torsors. This consists in defining a bijection between the set of rational points to be counted on $U$ and a certain set of integral points on an affine variety of higher dimension (which is equal to eight for quartic surfaces). Note that Derenthal has determined the equations of the universal torsors for most of the singular quartic del Pezzo surfaces in his doctoral thesis Der06a. This can also be achieved using only elementary techniques, see section 3 for an example.

Our aim is to prove Manin's conjecture for another surface split over $\mathbb{Q}$, having singularity type $\mathbf{A}_{3}$ and containing exactly four lines. This surface $V \subset \mathbb{P}^{4}$ is defined as the intersection of the two following quadrics,

$$
\begin{aligned}
x_{0} x_{1}-x_{2}^{2} & =0, \\
\left(x_{0}+x_{1}+x_{3}\right) x_{3}-x_{2} x_{4} & =0 .
\end{aligned}
$$

The lines on $V$ are given by $x_{i}=x_{2}=x_{3}=0$ and $x_{i}=x_{2}=x_{0}+x_{1}+x_{3}=0$ for $i \in\{0,1\}$ and the unique singularity is $(0: 0: 0: 0: 1)$. We see that $V$ is actually split over $\mathbb{Q}$ and thus, if $\widetilde{V}$ denotes the minimal desingularization of $V$, the Picard group of $\widetilde{V}$ has rank $\rho=6$. Define the open subset $U$ and the quantity $N_{U, H}(B)$ as explained above. In section 3 , we define a bijection between the set of the points to be counted on $U$ and a certain set of integral points of an open subset of the affine variety embedded in $\mathbb{A}^{10} \simeq \operatorname{Spec}\left(\mathbb{Q}\left[\eta_{1}, \ldots, \eta_{7}, \alpha_{1}, \alpha_{2}, \alpha_{4}\right]\right)$ and defined by

$$
\begin{aligned}
& \eta_{1}^{2} \eta_{2} \eta_{4}^{2} \eta_{7}+\eta_{5} \alpha_{1}-\eta_{6} \alpha_{2}=0, \\
& \eta_{2} \eta_{3}^{2} \eta_{5}^{2} \eta_{6}+\eta_{7} \alpha_{2}-\eta_{4} \alpha_{4}=0 .
\end{aligned}
$$

The universal torsor corresponding to our present problem actually has five equations and can be embedded in $\mathbb{A}^{11} \simeq \operatorname{Spec}\left(\mathbb{Q}\left[\eta_{1}, \ldots, \eta_{7}, \alpha_{1}, \alpha_{2}, \alpha_{3}, \alpha_{4}\right]\right)$ but we will neither use these three other equations nor the variable $\alpha_{3}$. Let us emphasize the fact that it is the first time that Manin's conjecture is proved for a split singular quartic del Pezzo surface whose universal torsor has several equations. This obstacle is overcome in section 5.1 by turning the two equations into a single congruence in order to apply the usual techniques. Our result is the following. 
Theorem 1. - As B tends to $+\infty$, we have the estimate

$$
N_{U, H}(B)=c_{V, H} B \log (B)^{5}\left(1+O\left(\frac{1}{\log (B)}\right)\right),
$$

where $c_{V, H}$ agrees with Peyre's prediction.

Since $\rho=6$, this estimate proves that $V$ satisfies Manin's conjecture. Let us note here that Derenthal has proved that $V$ is not toric [Der06b, Proposition 12] and Derenthal and Loughran have proved that it is not an equivariant compactification of $\mathbb{G}_{a}^{2}$ [DL10, so theorem 1 does not follow from the general results [BT98] and CLT02. In view of this result, it only remains to deal with five types of split singular quartic del Pezzo surfaces among the list of fifteen.

In the following section, we prove several lemmas about summations of arithmetic functions. The next two sections are respectively devoted to the calculations of the universal torsor and of Peyre's constant. Finally, the last section is dedicated to the proof of theorem [1]

It is a great pleasure for the author to thank his supervisor Professor de la Bretèche both for his encouragement and his advice during this work.

This work has received the financial support of the ANR PEPR (Points Entiers Points Rationnels).

\section{Arithmetic functions}

We need to introduce the following collection of arithmetic functions,

$$
\begin{aligned}
\varphi^{*}(n) & =\prod_{p \mid n}\left(1-\frac{1}{p}\right), & \varphi^{\circ}(n) & =\prod_{\substack{p \mid n \\
p \neq 2}}\left(1-\frac{1}{p-1}\right), \\
\varphi^{\dagger}(n) & =\prod_{p \mid n}\left(1-\frac{1}{p^{2}}\right), & \varphi^{b}(n) & =\prod_{\substack{p \mid n \\
p \neq 2}}\left(1+\frac{1}{p(p-2)}\right) .
\end{aligned}
$$

We can note here that if $n$ is odd then $\varphi^{\circ}(n) \varphi^{b}(n)=\varphi^{*}(n)$ and if $n$ is even then $\varphi^{\circ}(n) \varphi^{b}(n)=2 \varphi^{*}(n)$. Moreover, for $a, b \geq 1$, we define

$$
\psi_{a, b}(n)= \begin{cases}\varphi^{\circ}(\operatorname{gcd}(a, n))^{-1} & \text { if } \operatorname{gcd}(n, b)=1 \\ 0 & \text { otherwise }\end{cases}
$$

and

$$
\psi_{a, b}^{\prime}(n)= \begin{cases}\varphi^{\circ}(\operatorname{gcd}(a, n))^{-1} \varphi^{*}(n) \varphi^{*}(\operatorname{gcd}(a, n))^{-1} & \text { if } \operatorname{gcd}(n, b)=1 \\ 0 & \text { otherwise }\end{cases}
$$

Finally, for $\delta>0$, we set

$$
\sigma_{-\delta}(n)=\sum_{k \mid n} k^{-\delta}
$$

Lemma 1. - Let $0<\delta \leq 1$ be fixed. We have the estimate

$$
\sum_{n \leq X} \psi_{a, b}(n)=\Psi(a, b) X+O_{\delta}\left(\sigma_{-\delta}(a b) X^{\delta}\right)
$$

where

$$
\Psi(a, b)=\varphi^{*}(b) \frac{\varphi^{b}(a)}{\varphi^{b}(\operatorname{gcd}(a, b))}
$$


Proof. - We start by calculating the Dirichlet convolution of $\psi_{a, b}$ with the Möbius function $\mu$. We have

$$
\begin{aligned}
\left(\psi_{a, b} * \mu\right)(n) & =\sum_{d \mid n} \psi_{a, b}\left(\frac{n}{d}\right) \mu(d) \\
& =\prod_{p^{\nu} \| n}\left(\psi_{a, b}\left(p^{\nu}\right)-\psi_{a, b}\left(p^{\nu-1}\right)\right) .
\end{aligned}
$$

Moreover $\psi_{a, b}(1)=1$ and for all $\nu \geq 1$, we have

$$
\psi_{a, b}\left(p^{\nu}\right)=\psi_{a, b}(p)= \begin{cases}(1-1 /(p-1))^{-1} & \text { if } p \mid a, p \neq 2 \text { and } p \nmid b, \\ 1 & \text { if } p \neq 2, p \nmid a b, \\ 1 & \text { if } p=2,2 \nmid b, \\ 0 & \text { if } p \mid b .\end{cases}
$$

Thus, we easily obtain

$$
\left(\psi_{a, b} * \mu\right)(n)=\mu(n) \prod_{p \mid \operatorname{gcd}(a, n), p \nmid b} \frac{-1}{p-2},
$$

if $n \mid a b$ and $2 \nmid n$ or $2 \mid b$ and $\left(\psi_{a, b} * \mu\right)(n)=0$ otherwise. Writing $\psi_{a, b}=\left(\psi_{a, b} * \mu\right) * 1$, we get

$$
\begin{aligned}
\sum_{n \leq X} \psi_{a, b}(n) & =\sum_{n \leq X} \sum_{d \mid n}\left(\psi_{a, b} * \mu\right)(d) \\
& =\sum_{d=1}^{+\infty}\left(\psi_{a, b} * \mu\right)(d)\left[\frac{X}{d}\right] .
\end{aligned}
$$

Let $0<\delta \leq 1$ be fixed. Let us use the elementary estimate $[t]=t+O\left(t^{\delta}\right)$ for $t=X / d$. Since $\left|\left(\psi_{a, b} * \mu\right)(n)\right| \leq 1$, we get

$$
\sum_{d=1}^{+\infty} \frac{\left|\left(\psi_{a, b} * \mu\right)(d)\right|}{d^{\delta}} \leq \sigma_{-\delta}(a b)
$$

and we have thus proved that

$$
\sum_{n \leq X} \psi_{a, b}(n)=X \sum_{d=1}^{+\infty} \frac{\left(\psi_{a, b} * \mu\right)(d)}{d}+O\left(\sigma_{-\delta}(a b) X^{\delta}\right) .
$$

Finally, a straigthforward calculation gives

$$
\sum_{d=1}^{+\infty} \frac{\left(\psi_{a, b} * \mu\right)(d)}{d}=\prod_{p \mid b}\left(1-\frac{1}{p}\right) \prod_{\substack{p \mid a, p \nmid b \\ p \neq 2}}\left(1+\frac{1}{p(p-2)}\right)
$$

which concludes the proof.

Lemma 2. - Let $0<\delta \leq 1$ be fixed. We have the estimate

$$
\sum_{n \leq X} \psi_{a, b}^{\prime}(n)=\Psi^{\prime}(a, b) X+O_{\delta}\left(\sigma_{-\delta}(b) X^{\delta}\right),
$$

where

$$
\Psi^{\prime}(a, b)=\varphi^{*}(b) \frac{\varphi^{b}(a)}{\varphi^{b}(\operatorname{gcd}(a, b))} \frac{\zeta(2)^{-1}}{\varphi^{\dagger}(a b)}
$$


Proof. - We proceed exactly as for the proof of lemma 1, Let

$$
f(n)=\mu(n) \prod_{\substack{p \mid n, p \nmid a b \\ p \neq 2}} \frac{1}{p} \prod_{\substack{p \mid \operatorname{gcd}(a, n), p \nmid b \\ p \neq 2}} \frac{-1}{p-2} .
$$

A calculation provides

$$
\left(\psi_{a, b}^{\prime} * \mu\right)(n)= \begin{cases}f(n) & \text { if } 2 \nmid n \text { or } 2 \mid b, \\ f(n) / 2 & \text { if } 2 \mid n \text { and } 2 \nmid a b, \\ 0 & \text { otherwise. }\end{cases}
$$

Now we see that $\left|\left(\psi_{a, b}^{\prime} * \mu\right)(n)\right| \ll \operatorname{gcd}(b, n) / n$, which easily yields

$$
\sum_{d=1}^{+\infty} \frac{\left|\left(\psi_{a, b}^{\prime} * \mu\right)(d)\right|}{d^{\delta}} \ll \sigma_{-\delta}(b)
$$

Another straightforward calculation gives

$$
\sum_{d=1}^{+\infty} \frac{\left(\psi_{a, b}^{\prime} * \mu\right)(d)}{d}=\Psi^{\prime}(a, b)
$$

which completes the proof.

Using partial summation and the estimates of lemmas 1 and 2 as in the proof of [LB10, Lemma 6], we see that we have the following result.

Lemma 3. - Let $0<\delta \leq 1$ be fixed. Let $0 \leq t_{1}<t_{2}$ and $I=\left[t_{1}, t_{2}\right]$. Let also $g: \mathbb{R}_{>0} \rightarrow \mathbb{R}$ be a function having a piecewise continuous derivative on $I$ whose sign changes at most $R_{g}(I)$ times on $I$. We have

$$
\sum_{n \in I \cap \mathbb{Z}_{>0}} \psi_{a, b}(n) g(n)=\Psi(a, b) \int_{I} g(t) \mathrm{d} t+O_{\delta}\left(\sigma_{-\delta}(a b) t_{2}^{\delta} M_{I}(g)\right),
$$

and

$$
\sum_{n \in I \cap \mathbb{Z}_{>0}} \psi_{a, b}^{\prime}(n) g(n)=\Psi^{\prime}(a, b) \int_{I} g(t) \mathrm{d} t+O_{\delta}\left(\sigma_{-\delta}(b) t_{2}^{\delta} M_{I}(g)\right),
$$

where $M_{I}(g)=\left(1+R_{g}(I)\right) \sup _{t \in I \cap \mathbb{R}_{>0}}|g(t)|$.

We also have the following estimation.

Lemma 4. - With the same notations, if $2 \nmid b$ then

$$
\sum_{\substack{n \in I \cap \mathbb{Z}_{>0} \\ n \equiv 0(\bmod 2)}} \psi_{a, b}(n) g(n)=\frac{1}{2} \Psi(a, b) \int_{I} g(t) \mathrm{d} t+O_{\delta}\left(\sigma_{-\delta}(a b) t_{2}^{\delta} M_{I}(g)\right) .
$$

In a similar way, if $2 \mid a$ and $2 \nmid b$ then

$$
\sum_{\substack{n \in I \cap \mathbb{Z}_{>0} \\ n \equiv 0(\bmod 2)}} \psi_{a, b}^{\prime}(n) g(n)=\frac{1}{2} \Psi^{\prime}(a, b) \int_{I} g(t) \mathrm{d} t+O_{\delta}\left(\sigma_{-\delta}(b) t_{2}^{\delta} M_{I}(g)\right) .
$$


Proof. - Let us prove the statement for $\psi_{a, b}$, it suffices to notice that

$$
\begin{aligned}
\sum_{\substack{n \leq X \\
n \equiv 0(\bmod 2)}} \psi_{a, b}(n)= & \sum_{d=1}^{+\infty}\left(\psi_{a, b} * \mu\right)(d) \sum_{\substack{k \leq X / d \\
k \equiv 0(\bmod 2)}} 1 \\
& +\sum_{\substack{d=1 \\
d \equiv 0(\bmod 2)}}^{+\infty}\left(\psi_{a, b} * \mu\right)(d) \sum_{\substack{k \leq X / d \\
k \equiv 1(\bmod 2)}} 1,
\end{aligned}
$$

and $\left(\psi_{a, b} * \mu\right)(d)=0$ for all $d \equiv 0(\bmod 2)$ since $2 \nmid b$ and therefore

$$
\sum_{\substack{n \leq X \\ n \equiv 0(\bmod 2)}} \psi_{a, b}(n)=\sum_{d=1}^{+\infty}\left(\psi_{a, b} * \mu\right)(d)\left(\frac{X}{2 d}+O\left(\frac{X^{\delta}}{d^{\delta}}\right)\right) .
$$

We can conclude exactly as in the proof of lemma 1 and finally, as for lemma 3, use partial summation to complete the proof. The proof for $\psi_{a, b}^{\prime}$ is strictly identical, it only uses the fact that $\left(\psi_{a, b}^{\prime} * \mu\right)(d)=0$ for all $d \equiv 0(\bmod 2)$ since $2 \mid a$ and $2 \nmid b$.

\section{The universal torsor}

We now proceed to define a bijection between the set of rational points we want to count on $U$ and a certain set of integral points on the affine variety defined in the introduction. As explained in the introduction, the universal torsor of our problem is an open subset of an affine variety of dimension 8 embedded in $\mathbb{A}^{11}$. It has five equations but we will only deal with ten of the eleven variables and will only make use of two equations among these five. Our choice of notation might be surprising but it is guided by our wish to adopt the notation used by Derenthal in [Der06a, Chapter 6]. Note that if $\left(x_{0}: x_{1}: x_{2}: x_{3}: x_{4}\right) \in V(\mathbb{Q})$ then we have $\left(x_{0}: x_{1}: x_{2}: x_{3}: x_{4}\right) \in U(\mathbb{Q})$ if and only if $x_{0} x_{1} x_{2} x_{3} \neq 0$. Let $\left(x_{0}, x_{1}, x_{2}, x_{3}, x_{4}\right) \in \mathbb{Z}_{\neq 0}^{4} \times \mathbb{Z}$ be such that

$$
\begin{aligned}
x_{0} x_{1}-x_{2}^{2} & =0, \\
\left(x_{0}+x_{1}+x_{3}\right) x_{3}-x_{2} x_{4} & =0,
\end{aligned}
$$

and $\max \left\{\left|x_{i}\right|, 0 \leq i \leq 4\right\} \leq B$ and $\operatorname{gcd}\left(x_{0}, x_{1}, x_{2}, x_{3}, x_{4}\right)=1$. Since $\mathbf{x}=-\mathbf{x}$ in $\mathbb{P}^{4}$, we can assume that $x_{0}>0$, which implies $x_{1}>0$. Moreover, the symmetry given by $\left(x_{2}, x_{4}\right) \mapsto\left(-x_{2},-x_{4}\right)$ shows that we can also assume that $x_{2}>0$ keeping in mind that we need to multiply our future result by 2 . The first equation shows that there is a unique way to write $x_{0}=y_{01} x_{0}^{\prime 2}, x_{1}=y_{01} x_{1}^{\prime 2}$ and $x_{2}=y_{01} x_{0}^{\prime} x_{1}^{\prime}$ for some $x_{0}^{\prime}, x_{1}^{\prime}, y_{01}>0$ such that $\operatorname{gcd}\left(x_{0}^{\prime}, x_{1}^{\prime}\right)=1$. The second equation therefore gives

$$
\left(y_{01} x_{0}^{\prime 2}+y_{01} x_{1}^{\prime 2}+x_{3}\right) x_{3}-y_{01} x_{0}^{\prime} x_{1}^{\prime} x_{4}=0 .
$$

We define $y_{01}^{\prime}=\operatorname{gcd}\left(y_{01}, x_{3}\right)>0$ and write $y_{01}=y_{01}^{\prime} \eta_{2}$ and $x_{3}=y_{01}^{\prime} x_{3}^{\prime}$ with $\eta_{2}>0$ and $\operatorname{gcd}\left(\eta_{2}, x_{3}^{\prime}\right)=1$. We obtain

$$
\left(\eta_{2} x_{0}^{\prime 2}+\eta_{2} x_{1}^{\prime 2}+x_{3}^{\prime}\right) y_{01}^{\prime} x_{3}^{\prime}-\eta_{2} x_{0}^{\prime} x_{1}^{\prime} x_{4}=0,
$$

and thus $\eta_{2} \mid y_{01}^{\prime} x_{3}^{\prime 2}$ and it follows $\eta_{2} \mid y_{01}^{\prime}$ since $\operatorname{gcd}\left(\eta_{2}, x_{3}^{\prime}\right)=1$. We can therefore write $y_{01}^{\prime}=\eta_{2} y_{01}^{\prime \prime}$ for some $y_{01}^{\prime \prime}>0$. The equation becomes

$$
\left(\eta_{2} x_{0}^{\prime 2}+\eta_{2} x_{1}^{\prime 2}+x_{3}^{\prime}\right) y_{01}^{\prime \prime} x_{3}^{\prime}-x_{0}^{\prime} x_{1}^{\prime} x_{4}=0 .
$$

We now see that $\operatorname{gcd}\left(x_{0}, x_{1}, x_{2}, x_{3}, x_{4}\right)=1$ implies $\operatorname{gcd}\left(y_{01}^{\prime \prime}, x_{4}\right)=1$ and thus $y_{01}^{\prime \prime} \mid x_{0}^{\prime} x_{1}^{\prime}$ and $x_{0}^{\prime}, x_{1}^{\prime}$ being coprime, we can write $y_{01}^{\prime \prime}=\eta_{1} \eta_{3}, x_{0}^{\prime}=\eta_{3} x_{0}^{\prime \prime}$ and $x_{1}^{\prime}=\eta_{1} x_{1}^{\prime \prime}$ for some 
$\eta_{1}, \eta_{3}, x_{0}^{\prime \prime}, x_{1}^{\prime \prime}>0$. Now we set $x_{3}^{\prime}=\alpha_{1} x_{3}^{\prime \prime}, x_{4}=\alpha_{1} \alpha_{4}$ with $x_{3}^{\prime \prime}>0$ and $\operatorname{gcd}\left(x_{3}^{\prime \prime}, \alpha_{4}\right)=1$ (we do not prescribe the sign of $\alpha_{1}= \pm \operatorname{gcd}\left(x_{3}^{\prime}, x_{4}\right)$ ). We finally get

$$
\left(\eta_{2} \eta_{3}^{2} x_{0}^{\prime \prime 2}+\eta_{2} \eta_{1}^{2} x_{1}^{\prime \prime 2}+\alpha_{1} x_{3}^{\prime \prime}\right) x_{3}^{\prime \prime}-x_{0}^{\prime \prime} x_{1}^{\prime \prime} \alpha_{4}=0
$$

We observe that since $\operatorname{gcd}\left(x_{3}^{\prime \prime}, \alpha_{4}\right)=1$, we have $x_{3}^{\prime \prime} \mid x_{0}^{\prime \prime} x_{1}^{\prime \prime}$ and we can write $x_{3}^{\prime \prime}=\eta_{5} \eta_{7}$, $x_{0}^{\prime \prime}=\eta_{5} \eta_{6}$ and $x_{1}^{\prime \prime}=\eta_{4} \eta_{7}$, for some $\eta_{4}, \eta_{5}, \eta_{6}, \eta_{7}>0$. We have finally obtained

$$
\begin{aligned}
x_{0} & =\eta_{1} \eta_{2}^{2} \eta_{3}^{3} \eta_{5}^{2} \eta_{6}^{2}, \\
x_{1} & =\eta_{1}^{3} \eta_{2}^{2} \eta_{3} \eta_{4}^{2} \eta_{7}^{2}, \\
x_{2} & =\eta_{1}^{2} \eta_{2}^{2} \eta_{3}^{2} \eta_{4} \eta_{5} \eta_{6} \eta_{7}, \\
x_{3} & =\eta_{1} \eta_{2} \eta_{3} \eta_{5} \eta_{7} \alpha_{1}, \\
x_{4} & =\alpha_{1} \alpha_{4},
\end{aligned}
$$

and the equation is

$$
\eta_{2} \eta_{3}^{2} \eta_{5}^{2} \eta_{6}^{2}+\eta_{1}^{2} \eta_{2} \eta_{4}^{2} \eta_{7}^{2}+\eta_{5} \eta_{7} \alpha_{1}-\eta_{4} \eta_{6} \alpha_{4}=0
$$

Furthermore, it is easy to see that the coprimality conditions can be summed up by

$$
\begin{aligned}
& \operatorname{gcd}\left(\eta_{3} \eta_{5} \eta_{6}, \eta_{1} \eta_{4} \eta_{7}\right)=1, \\
& \operatorname{gcd}\left(\eta_{5} \eta_{7}, \eta_{2} \alpha_{4}\right)=1, \\
& \operatorname{gcd}\left(\eta_{1} \eta_{2} \eta_{3}, \alpha_{1} \alpha_{4}\right)=1
\end{aligned}
$$

Since $\eta_{6}$ and $\eta_{7}$ are coprime, we see that the equation is equivalent to the existence of $\alpha_{2} \in \mathbb{Z}$ such that

$$
\begin{aligned}
& \eta_{1}^{2} \eta_{2} \eta_{4}^{2} \eta_{7}+\eta_{5} \alpha_{1}-\eta_{6} \alpha_{2}=0 \\
& \eta_{2} \eta_{3}^{2} \eta_{5}^{2} \eta_{6}+\eta_{7} \alpha_{2}-\eta_{4} \alpha_{4}=0
\end{aligned}
$$

In a similar way, since $\eta_{4}$ and $\eta_{5}$ are coprime, we can derive the existence of $\alpha_{3} \in \mathbb{Z}$ such that

$$
\begin{aligned}
\eta_{2} \eta_{3}^{2} \eta_{5} \eta_{6}^{2}+\eta_{7} \alpha_{1}-\eta_{4} \alpha_{3} & =0 \\
\eta_{1}^{2} \eta_{2} \eta_{4} \eta_{7}^{2}+\eta_{5} \alpha_{3}-\eta_{6} \alpha_{4} & =0 \\
\eta_{1}^{2} \eta_{2}^{2} \eta_{3}^{2} \eta_{4} \eta_{5} \eta_{6} \eta_{7}+\alpha_{1} \alpha_{4}-\alpha_{2} \alpha_{3} & =0 .
\end{aligned}
$$

As explained above, we will not use these three equations. We define $\mathcal{T}(B)$ as the set of $\left(\eta_{1}, \eta_{2}, \eta_{3}, \eta_{4}, \eta_{5}, \eta_{6}, \eta_{7}, \alpha_{1}, \alpha_{2}, \alpha_{4}\right) \in \mathbb{Z}_{>0}^{7} \times \mathbb{Z}^{3}$ satisfying the coprimality conditions (3.1), (3.2), (3.3), the two equations (3.4) and (3.5) and finally the height conditions

$$
\begin{aligned}
\eta_{1} \eta_{2}^{2} \eta_{3}^{3} \eta_{5}^{2} \eta_{6}^{2} & \leq B \\
\eta_{1}^{3} \eta_{2}^{2} \eta_{3} \eta_{4}^{2} \eta_{7}^{2} & \leq B \\
\eta_{1} \eta_{2} \eta_{3} \eta_{5} \eta_{7}\left|\alpha_{1}\right| & \leq B \\
\left|\alpha_{1} \alpha_{4}\right| & \leq B .
\end{aligned}
$$

We have proved the following lemma.

Lemma 5. - We have the equality

$$
N_{U, H}(B)=2 \# \mathcal{T}(B) .
$$




\section{Calculation of Peyre's constant}

We calculate the value of the constant $c_{V, H}$ predicted by Peyre. It is defined by

$$
c_{V, H}=\alpha(\widetilde{V}) \beta(\widetilde{V}) \omega_{H}(\widetilde{V}),
$$

where $\alpha(\widetilde{V}) \in \mathbb{Q}$ is the volume of a certain polytope in the dual of the effective cone of $\widetilde{V}$ with respect to the intersection form, $\beta(\tilde{V})=\# H^{1}\left(\operatorname{Gal}(\overline{\mathbb{Q}} / \mathbb{Q}), \operatorname{Pic}_{\overline{\mathbb{Q}}}(\widetilde{V})\right)=1$ since $V$ is split over $\mathbb{Q}$ and finally

$$
\omega_{H}(\widetilde{V})=\omega_{\infty} \prod_{p}\left(1-\frac{1}{p}\right)^{6} \omega_{p}
$$

where $\omega_{\infty}$ and $\omega_{p}$ are respectively the archimedean and $p$-adic densities. The work of Derenthal [Der07] provides the value

$$
\alpha(\widetilde{V})=\frac{1}{4320} .
$$

Furthermore, using Lou10, Lemma 2.3], we get

$$
\omega_{p}=1+\frac{6}{p}+\frac{1}{p^{2}} .
$$

To calculate $\omega_{\infty}$, we set $f_{1}(x)=x_{0} x_{1}-x_{2}^{2}, f_{2}(x)=\left(x_{0}+x_{1}+x_{3}\right) x_{3}-x_{2} x_{4}$ and we parametrize the points of $V$ by $x_{0}, x_{2}$ and $x_{3}$. We have

$$
\begin{aligned}
\operatorname{det}\left(\begin{array}{rl}
\frac{\partial f_{1}}{\partial x_{1}} & \frac{\partial f_{1}}{\partial x_{4}} \\
\frac{\partial f_{2}}{\partial x_{1}} & \frac{\partial f_{2}}{\partial x_{4}}
\end{array}\right) & =\left|\begin{array}{cc}
x_{0} & 0 \\
x_{3} & -x_{2}
\end{array}\right| \\
& =-x_{0} x_{2} .
\end{aligned}
$$

Moreover, $x_{1}=x_{2}^{2} / x_{0}$ and $x_{4}=\left(x_{0}^{2}+x_{2}^{2}+x_{0} x_{3}\right) x_{3} /\left(x_{0} x_{2}\right)$. Since $\mathbf{x}=-\mathbf{x}$ in $\mathbb{P}^{4}$, we have

$$
\omega_{\infty}=2 \iiint_{x_{0}, x_{2}>0, x_{0}, x_{2}^{2} / x_{0},\left|x_{3}\right|,\left|x_{0}^{2}+x_{2}^{2}+x_{0} x_{3}\right|\left|x_{3}\right| / x_{0} x_{2} \leq 1} \frac{\mathrm{d} x_{0} \mathrm{~d} x_{2} \mathrm{~d} x_{3}}{x_{0} x_{2}} .
$$

Define the function

$$
h:\left(u_{2}, t_{7}, t_{6}\right) \mapsto \max \left\{t_{6}, t_{7}, t_{7}\left|t_{7}-t_{6} u_{2}\right|,\left|t_{7}-t_{6} u_{2}\right|\left|t_{6}+t_{7} u_{2}\right|\right\} .
$$

The change of variables given by $x_{0}=t_{6}^{2}, x_{2}=t_{6} t_{7}$ and $x_{3}=-t_{7}\left(t_{7}-t_{6} u_{2}\right)$ yields

$$
\omega_{\infty}=4 \iiint_{t_{6}, t_{7}>0, h\left(u_{2}, t_{7}, t_{6}\right) \leq 1} \mathrm{~d} u_{2} \mathrm{~d} t_{7} \mathrm{~d} t_{6}
$$

\section{Proof of the main theorem}

5.1. First steps of the proof. - The idea of the proof is to see the equations (3.4) and (3.5) as congruences respectively modulo $\eta_{5}$ and $\eta_{4}$ and then to count the number of $\alpha_{2}$ satisfying these two congruences. In order to do so, we replace the height conditions (3.8) and (3.9) by

$$
\begin{aligned}
\eta_{1} \eta_{2} \eta_{3} \eta_{7}\left|\eta_{1}^{2} \eta_{2} \eta_{4}^{2} \eta_{7}-\eta_{6} \alpha_{2}\right| & \leq B \\
\eta_{4}^{-1} \eta_{5}^{-1}\left|\eta_{1}^{2} \eta_{2} \eta_{4}^{2} \eta_{7}-\eta_{6} \alpha_{2}\right|\left|\eta_{2} \eta_{3}^{2} \eta_{5}^{2} \eta_{6}+\eta_{7} \alpha_{2}\right| & \leq B
\end{aligned}
$$

and we carry on denoting them the same way. We note that the equation (3.4) proves that we necessarily have $\operatorname{gcd}\left(\eta_{1} \eta_{2}, \eta_{6} \alpha_{2}\right)=1$ since we also have $\operatorname{gcd}\left(\eta_{1} \eta_{2}, \eta_{5} \alpha_{1}\right)=1$. Exactly the same way we get $\operatorname{gcd}\left(\alpha_{2}, \eta_{3} \eta_{5}\right)=1$ thanks to the equation (3.5) and $\operatorname{gcd}\left(\eta_{3} \eta_{5}, \eta_{4} \alpha_{4}\right)=1$. The equation (3.5) and $\operatorname{gcd}\left(\eta_{2}, \eta_{7} \alpha_{2}\right)=1$ also imply $\operatorname{gcd}\left(\eta_{2}, \eta_{4}\right)=1$. This new coprimality condition together with the equation (3.5) 
yield $\operatorname{gcd}\left(\eta_{4}, \alpha_{2}\right)=1$ since we have $\operatorname{gcd}\left(\eta_{4}, \eta_{2} \eta_{3} \eta_{5} \eta_{6}\right)=1$. In a similar way, we finally obtain $\operatorname{gcd}\left(\alpha_{1}, \eta_{4} \eta_{6}\right)=1, \operatorname{gcd}\left(\eta_{4}, \eta_{7}\right)=1$ and $\operatorname{gcd}\left(\eta_{5}, \eta_{6}\right)=1$. We can therefore rewrite the coprimality conditions (3.1), (3.2), (3.3) and all these new conditions as

$$
\begin{aligned}
& \operatorname{gcd}\left(\alpha_{1}, \eta_{1} \eta_{2} \eta_{3} \eta_{4} \eta_{6}\right)=1, \\
& \operatorname{gcd}\left(\alpha_{4}, \eta_{1} \eta_{2} \eta_{3} \eta_{5} \eta_{7}\right)=1, \\
& \operatorname{gcd}\left(\alpha_{2}, \eta_{1} \eta_{2} \eta_{3} \eta_{4} \eta_{5}\right)=1, \\
& \operatorname{gcd}\left(\eta_{7}, \eta_{2} \eta_{3} \eta_{4} \eta_{5} \eta_{6}\right)=1, \\
& \operatorname{gcd}\left(\eta_{6}, \eta_{1} \eta_{2} \eta_{4} \eta_{5}\right)=1, \\
& \operatorname{gcd}\left(\eta_{1} \eta_{4}, \eta_{3} \eta_{5}\right)=1, \\
& \operatorname{gcd}\left(\eta_{2}, \eta_{4} \eta_{5}\right)=1 .
\end{aligned}
$$

From now on, we set $\boldsymbol{\eta}=\left(\eta_{1}, \eta_{2}, \eta_{3}, \eta_{4}, \eta_{5}\right) \in \mathbb{Z}_{>0}^{5}$ and $\boldsymbol{\eta}^{\prime}=\left(\boldsymbol{\eta}, \eta_{6}, \eta_{7}\right) \in \mathbb{Z}_{>0}^{7}$. Consider that $\boldsymbol{\eta}^{\prime} \in \mathbb{Z}_{>0}^{7}$ is fixed and is subject to the height conditions (3.6), (3.7) and to the coprimality conditions (5.4), (5.5), (5.6) and (5.7). Let $N\left(\boldsymbol{\eta}^{\prime}, B\right)$ be the number of $\left(\alpha_{1}, \alpha_{2}, \alpha_{4}\right) \in \mathbb{Z}$ satisfying the equations (3.4), (3.5), the height conditions (3.8) and (3.9) and finally the coprimality conditions (5.1), (5.2) and (5.3). For $\left(r_{1}, r_{2}, r_{3}, r_{4}, r_{5}\right) \in \mathbb{Q}^{5}$, we define

$$
\boldsymbol{\eta}^{\left(r_{1}, r_{2}, r_{3}, r_{4}, r_{5}\right)}=\eta_{1}^{r_{1}} \eta_{2}^{r_{2}} \eta_{3}^{r_{3}} \eta_{4}^{r_{4}} \eta_{5}^{r_{5}},
$$

and we adopt the following notations in order to help in the understanding of the height conditions,

$$
\begin{aligned}
A_{2} & =\boldsymbol{\eta}^{(1,1,1,1,1)} \\
Y_{6} & =\frac{B^{1 / 2}}{\boldsymbol{\eta}^{(1 / 2,1,3 / 2,0,1)}} \\
Y_{7} & =\frac{B^{1 / 2}}{\boldsymbol{\eta}^{(3 / 2,1,1 / 2,1,0)}}
\end{aligned}
$$

and recalling the definition (4.1) of the function $h$, we can sum up the height conditions (3.6), (3.7), (3.8) and (3.9) as

$$
h\left(\frac{\alpha_{2}}{A_{2}}, \frac{\eta_{7}}{Y_{7}}, \frac{\eta_{6}}{Y_{6}}\right) \leq 1 .
$$

We also introduce the real-valued functions

$$
\begin{array}{ccc}
g_{1}:\left(t_{7}, t_{6}\right) \mapsto \int_{h\left(u_{2}, t_{7}, t_{6}\right) \leq 1} \mathrm{~d} u_{2}, \\
g_{2}: \quad\left(t_{6} ; \boldsymbol{\eta}, B\right) \mapsto \int_{t_{7} Y_{7} \geq 1} g_{1}\left(t_{7}, t_{6}\right) \mathrm{d} t_{7}, \\
g_{3} \quad: \quad(\boldsymbol{\eta}, B) \mapsto \int_{t_{6} Y_{6} \geq 1} g_{2}\left(t_{6} ; \boldsymbol{\eta}, B\right) \mathrm{d} t_{6} .
\end{array}
$$

We obviously have

$$
g_{3}(\boldsymbol{\eta}, B)=\iiint_{t_{6} Y_{6} \geq 1, t_{7} Y_{7} \geq 1, h\left(u_{2}, t_{7}, t_{6}\right) \leq 1} \mathrm{~d} u_{2} \mathrm{~d} t_{7} \mathrm{~d} t_{6} .
$$

Lemma 6. - We have the bounds

$$
\begin{aligned}
g_{1}\left(t_{7}, t_{6}\right) & \ll t_{6}^{-1 / 2} t_{7}^{-1 / 2}, \\
g_{2}\left(t_{6} ; \boldsymbol{\eta}, B\right) & \ll t_{6}^{-1 / 2} .
\end{aligned}
$$


Proof. - Recall the definition (4.1) of the function $h$. A little thought reveals that the condition $\left|t_{7}-t_{6} u_{2}\right|\left|t_{6}+t_{7} u_{2}\right| \leq 1$ implies that $u_{2}$ runs over a set whose measure is $\ll t_{6}^{-1 / 2} t_{7}^{-1 / 2}$ which gives the first bound. The second bound is an immediate consequence of the first since $t_{7} \leq 1$.

We have the following result.

Lemma 7. - The following estimate holds

$$
N\left(\boldsymbol{\eta}^{\prime}, B\right)=\frac{A_{2}}{\eta_{4} \eta_{5}} g_{1}\left(\frac{\eta_{7}}{Y_{7}}, \frac{\eta_{6}}{Y_{6}}\right) \theta\left(\boldsymbol{\eta}^{\prime}\right)+R\left(\boldsymbol{\eta}^{\prime}, B\right),
$$

where $\theta\left(\boldsymbol{\eta}^{\prime}\right)$ is a certain arithmetic function given in (5.9) and

$$
\sum_{\boldsymbol{\eta}^{\prime}} R\left(\boldsymbol{\eta}^{\prime}, B\right) \ll B \log (B)^{2} .
$$

Let us remove the coprimality conditions (5.1) and (5.2) employing two Möbius inversions, we get

$$
N\left(\boldsymbol{\eta}^{\prime}, B\right)=\sum_{k_{1} \mid \eta_{1} \eta_{2} \eta_{3} \eta_{4} \eta_{6}} \mu\left(k_{1}\right) \sum_{k_{4} \mid \eta_{1} \eta_{2} \eta_{3} \eta_{5} \eta_{7}} \mu\left(k_{4}\right) S_{k_{1}, k_{4}},
$$

where, with the notations $\alpha_{1}=k_{1} \alpha_{1}^{\prime}$ and $\alpha_{4}=k_{4} \alpha_{4}^{\prime}$,

$$
\begin{aligned}
& S_{k_{1}, k_{4}}=\#\left\{\begin{array}{ll}
\left.\alpha_{1}^{\prime}, \alpha_{4}^{\prime}, \alpha_{2}\right) \in \mathbb{Z}^{3}, & \eta_{2} \eta_{3}^{2} \eta_{4}^{2} \eta_{7} \eta_{6}+\eta_{5} k_{1} \alpha_{1}^{\prime}-\eta_{6} \alpha_{2}=0 \\
& (3.8), \eta_{4} k_{4} \alpha_{4}^{\prime}=0
\end{array}\right\} \\
& =\#\left\{\begin{array}{ll}
\eta_{6} \alpha_{2} \equiv \eta_{1}^{2} \eta_{2} \eta_{4}^{2} \eta_{7}\left(\bmod k_{1} \eta_{5}\right) \\
\alpha_{2} \in \mathbb{Z}, & \eta_{7} \alpha_{2} \equiv-\eta_{2} \eta_{3}^{2} \eta_{5}^{2} \eta_{6}\left(\bmod k_{4} \eta_{4}\right) \\
& (3.8),(3.9),(5.3)
\end{array}\right\} .
\end{aligned}
$$

We note that we necessarily have $\operatorname{gcd}\left(k_{1}, \eta_{6}\right)=1 \operatorname{since} \operatorname{gcd}\left(\eta_{6}, \eta_{1} \eta_{2} \eta_{4} \eta_{7}\right)=1$ and $\operatorname{gcd}\left(k_{1}, \eta_{1} \eta_{2} \eta_{4}\right)=1$ since $\operatorname{gcd}\left(\eta_{1} \eta_{2} \eta_{4}, \eta_{6} \alpha_{2}\right)=1$. In a similar way, we also have $\operatorname{gcd}\left(k_{4}, \eta_{2} \eta_{3} \eta_{5} \eta_{7}\right)=1$. In particular, we see that $\eta_{6}$ and $\eta_{7}$ are respectively invertible modulo $k_{1} \eta_{5}$ and $k_{4} \eta_{4}$. We therefore get

$$
N\left(\boldsymbol{\eta}^{\prime}, B\right)=\sum_{\substack{k_{1} \mid \eta_{3} \\ \operatorname{gcd}\left(k_{1}, \eta_{1} \eta_{2} \eta_{4} \eta_{6}\right)=1}} \mu\left(k_{1}\right) \sum_{\substack{k_{4} \mid \eta_{1} \\ \operatorname{gcd}\left(k_{4}, \eta_{2} \eta_{3} \eta_{5} \eta_{7}\right)=1}} \mu\left(k_{4}\right) S_{k_{1}, k_{4}},
$$

and

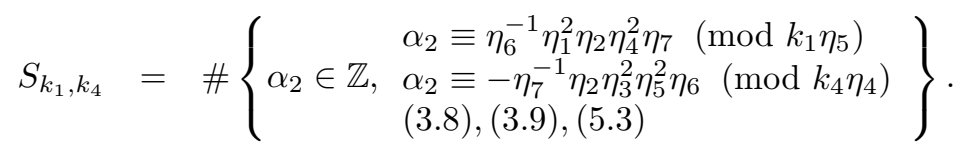

Furthermore, $k_{1} \eta_{5}$ and $k_{4} \eta_{4}$ are coprime since $\eta_{3} \eta_{5}$ and $\eta_{1} \eta_{4}$ are coprime thus the Chinese remainder theorem gives

$$
S_{k_{1}, k_{4}}=\#\left\{\alpha_{2} \in \mathbb{Z}, \begin{array}{l}
\alpha_{2} \equiv a\left(\bmod k_{1} k_{4} \eta_{4} \eta_{5}\right) \\
(3.8),(3.9),(15.3)
\end{array}\right\},
$$

for a certain integer $a$ coprime to $k_{1} k_{4} \eta_{4} \eta_{5}$ since $\operatorname{gcd}\left(k_{1} k_{4} \eta_{4} \eta_{5}, \alpha_{2}\right)=1$. A Möbius inversion yields

$$
\begin{aligned}
& S_{k_{1}, k_{4}}=\sum_{k_{2} \mid \eta_{1} \eta_{2} \eta_{3} \eta_{4} \eta_{5}} \mu\left(k_{2}\right) \#\left\{\alpha_{2}^{\prime} \in \mathbb{Z}, \begin{array}{l}
k_{2} \alpha_{2}^{\prime} \equiv a\left(\bmod k_{1} k_{4} \eta_{4} \eta_{5}\right) \\
(3.8),(3.9)
\end{array}\right\} \\
& =\sum_{\substack{k_{2} \mid \eta_{1} \eta_{2} \eta_{3} \\
\operatorname{gcd}\left(k_{2}, k_{1} k_{4} \eta_{4} \eta_{5}\right)=1}} \mu\left(k_{2}\right) \#\left\{\alpha_{2}^{\prime} \in \mathbb{Z}, \begin{array}{l}
\alpha_{2}^{\prime} \equiv k_{2}^{-1} a\left(\bmod k_{1} k_{4} \eta_{4} \eta_{5}\right) \\
(\underline{3.9})
\end{array}\right\},
\end{aligned}
$$


since $\operatorname{gcd}\left(k_{1} k_{4} \eta_{4} \eta_{5}, a\right)=1$. Using the elementary estimate

$$
\#\left\{n \in \mathbb{Z} \cap\left[t_{1}, t_{2}\right], n \equiv a(\bmod q)\right\}=\frac{t_{2}-t_{1}}{q}+O(1),
$$

and the change of variable $u_{2} \mapsto u_{2} A_{2} / k_{2}$, we get

$$
\#\left\{\alpha_{2}^{\prime} \in \mathbb{Z}, \begin{array}{l}
\alpha_{2}^{\prime} \equiv k_{2}^{-1} a \\
(3.8),\left(\bmod k_{1}\right)
\end{array}\left(k_{1} k_{4} \eta_{4} \eta_{5}\right)\right\}=\frac{A_{2}}{k_{2} k_{1} k_{4} \eta_{4} \eta_{5}} g_{1}\left(\frac{\eta_{7}}{Y_{7}}, \frac{\eta_{6}}{Y_{6}}\right)+O(1) .
$$

We see that the main term of $N\left(\boldsymbol{\eta}^{\prime}, B\right)$ is equal to

$$
\frac{A_{2}}{\eta_{4} \eta_{5}} g_{1}\left(\frac{\eta_{7}}{Y_{7}}, \frac{\eta_{6}}{Y_{6}}\right) \theta\left(\boldsymbol{\eta}^{\prime}\right)
$$

where

$$
\begin{aligned}
\theta\left(\boldsymbol{\eta}^{\prime}\right) & =\sum_{\substack{k_{1} \mid \eta_{3} \\
\operatorname{gcd}\left(k_{1}, \eta_{1} \eta_{2} \eta_{4} \eta_{6}\right)=1}} \frac{\mu\left(k_{1}\right)}{k_{1}} \sum_{\substack{k_{4} \mid \eta_{1} \\
\operatorname{gcd}\left(k_{4}, \eta_{2} \eta_{3} \eta_{5} \eta_{7}\right)=1}} \frac{\mu\left(k_{4}\right)}{k_{4}} \sum_{\substack{\operatorname{gcd}\left(k_{2}, k_{1} k_{4} \eta_{4} \eta_{5}\right)=1 \\
k_{2} \eta_{2} \eta_{3}}} \frac{\mu\left(k_{2}\right)}{k_{2}} \\
& =\varphi^{*}\left(\eta_{1} \eta_{2} \eta_{3} \eta_{4} \eta_{5}\right) \sum_{\substack{k_{1} \mid \eta_{3} \\
\operatorname{gcd}\left(k_{1}, \eta_{2} \eta_{6}\right)=1}} \frac{\mu\left(k_{1}\right)}{k_{1} \varphi^{*}\left(k_{1} \eta_{5}\right)} \sum_{\substack{k_{4} \mid \eta_{1} \\
\operatorname{gcd}\left(k_{4}, \eta_{2} \eta_{7}\right)=1}} \frac{\mu\left(k_{4}\right)}{k_{4} \varphi^{*}\left(k_{4} \eta_{4}\right)} .
\end{aligned}
$$

We have removed $\eta_{1} \eta_{4}$ from the condition over $k_{1}$ and $\eta_{3} \eta_{5}$ from the condition over $k_{4}$ respectively because $\operatorname{gcd}\left(\eta_{3}, \eta_{1} \eta_{4}\right)=1$ and $\operatorname{gcd}\left(\eta_{1}, \eta_{3} \eta_{5}\right)=1$. A straightforward calculation yields, for $a, b, c \geq 1$,

$$
\sum_{\substack{k \mid a \\ \operatorname{gcd}(k, c)=1}} \frac{\mu(k)}{k \varphi^{*}(k b)}=\frac{\varphi^{*}(\operatorname{gcd}(a, b))}{\varphi^{*}(b) \varphi^{*}(\operatorname{gcd}(a, b, c))} \prod_{p \mid a, p \nmid b c}\left(1-\frac{1}{p-1}\right) .
$$

Therefore, we have obtained

$$
\theta\left(\boldsymbol{\eta}^{\prime}\right)=\theta_{1}\left(\boldsymbol{\eta}, \eta_{6}\right) \prod_{p \mid \eta_{1}, p \nmid \eta_{2} \eta_{4} \eta_{7}}\left(1-\frac{1}{p-1}\right),
$$

where $\theta_{1}\left(\boldsymbol{\eta}, \eta_{6}\right)$ denotes

$$
\varphi^{*}\left(\eta_{1} \eta_{2} \eta_{3} \eta_{4} \eta_{5}\right) \frac{\varphi^{*}\left(\operatorname{gcd}\left(\eta_{1}, \eta_{4}\right)\right)}{\varphi^{*}\left(\eta_{4}\right)} \frac{\varphi^{*}\left(\operatorname{gcd}\left(\eta_{3}, \eta_{5}\right)\right)}{\varphi^{*}\left(\eta_{5}\right)} \prod_{p \mid \eta_{3}, p \nmid \eta_{2} \eta_{5} \eta_{6}}\left(1-\frac{1}{p-1}\right) .
$$

In addition, we see that the overall contribution of the error term is

$$
\begin{aligned}
\sum_{\boldsymbol{\eta}, \eta_{6}, \eta_{7}} 2^{\omega\left(\eta_{3}\right)} 2^{\omega\left(\eta_{1}\right)} 2^{\omega\left(\eta_{1} \eta_{2} \eta_{3}\right)} & \ll \sum_{\boldsymbol{\eta}} 2^{\omega\left(\eta_{3}\right)} 2^{\omega\left(\eta_{1}\right)} 2^{\omega\left(\eta_{1} \eta_{2} \eta_{3}\right)} Y_{6} Y_{7} \\
& =\sum_{\boldsymbol{\eta}} 2^{\omega\left(\eta_{3}\right)} 2^{\omega\left(\eta_{1}\right)} 2^{\omega\left(\eta_{1} \eta_{2} \eta_{3}\right)} \frac{B}{\boldsymbol{\eta}^{(2,2,2,1,1)}} \\
& \ll B \log (B)^{2},
\end{aligned}
$$

where we have summed over $\eta_{6}$ and $\eta_{7}$ using respectively the height conditions (3.6) and (3.7). This completes the proof of lemma 7 
5.2. Summation over $\eta_{7}$. - To carry out the summations over $\eta_{7}$ and $\eta_{6}$, we let

$$
\mathcal{V}=\left\{\boldsymbol{\eta} \in \mathbb{Z}_{>0}^{5}, Y_{6} \geq 1, Y_{7} \geq 1\right\},
$$

and we assume that $\boldsymbol{\eta} \in \mathcal{V}$ is fixed and is subject to the coprimality conditions (5.6) and (5.7). Our next task is to sum over $\eta_{7}$, that is why we have isolated $\eta_{7}$ in $\theta\left(\boldsymbol{\eta}^{\prime}\right)$. Let us define

$$
\mathcal{N}=\left\{\left(\eta_{1}, \eta_{2}, \eta_{4}\right) \in \mathbb{Z}_{>0}^{3}, 2 \nmid \eta_{1} \text { or } 2 \mid \eta_{2} \eta_{4}\right\} .
$$

It is plain to see that if $\left(\eta_{1}, \eta_{2}, \eta_{4}\right) \in \mathcal{N}$ or $2 \mid \eta_{7}$ then

$$
\prod_{p \mid \eta_{1}, p \nmid \eta_{2} \eta_{4} \eta_{7}}\left(1-\frac{1}{p-1}\right)=\prod_{\substack{p \mid \eta_{1}, p \nmid \eta_{2} \eta_{4} \eta_{7} \\ p \neq 2}}\left(1-\frac{1}{p-1}\right),
$$

and this product is equal to 0 otherwise. Furthermore, since $\eta_{2} \eta_{4}$ and $\eta_{7}$ are coprime, we see that

$$
\prod_{\substack{p \mid \eta_{1}, p \nmid \eta_{2} \eta_{4} \eta_{7} \\ p \neq 2}}\left(1-\frac{1}{p-1}\right)=\frac{\varphi^{\circ}\left(\eta_{1}\right)}{\varphi^{\circ}\left(\operatorname{gcd}\left(\eta_{1}, \eta_{2} \eta_{4}\right)\right) \varphi^{\circ}\left(\operatorname{gcd}\left(\eta_{1}, \eta_{7}\right)\right)} .
$$

We need to treat two cases separately depending on whether $\left(\eta_{1}, \eta_{2}, \eta_{4}\right) \in \mathcal{N}$ or $\left(\eta_{1}, \eta_{2}, \eta_{4}\right) \notin \mathcal{N}$ (note that, in the latter case, the main term of $N\left(\boldsymbol{\eta}^{\prime}, B\right)$ vanishes if $2 \nmid \eta_{7}$ ). For fixed $\eta_{6}$ satisfying the height condition (3.6) and the coprimality condition (5.5), we call $N\left(\boldsymbol{\eta}, \eta_{6}, B\right)$ the sum of the main term of $N\left(\boldsymbol{\eta}^{\prime}, B\right)$ over $\eta_{7}$, $\eta_{7}$ being subject to the height condition (3.7) and to the coprimality condition (5.4). We also use $N_{1}\left(\boldsymbol{\eta}, \eta_{6}, B\right)$ and $N_{2}\left(\boldsymbol{\eta}, \eta_{6}, B\right)$ to denote the sums over $\eta_{7}$ respectively for $\left(\eta_{1}, \eta_{2}, \eta_{4}\right) \in \mathcal{N}$ and $\left(\eta_{1}, \eta_{2}, \eta_{4}\right) \notin \mathcal{N}$. We now proceed to prove the following lemma.

Lemma 8. - We have the estimate

$$
N\left(\boldsymbol{\eta}, \eta_{6}, B\right)=\frac{A_{2} Y_{7}}{\eta_{4} \eta_{5}} g_{2}\left(\frac{\eta_{6}}{Y_{6}} ; \boldsymbol{\eta}, B\right) \theta_{1}^{\prime}(\boldsymbol{\eta}) \theta_{2}^{\prime}\left(\boldsymbol{\eta}, \eta_{6}\right)+R\left(\boldsymbol{\eta}, \eta_{6}, B\right),
$$

where $\theta_{1}^{\prime}(\boldsymbol{\eta})$ and $\theta_{2}^{\prime}\left(\boldsymbol{\eta}, \eta_{6}\right)$ are arithmetic functions defined in (5.11) and (5.12) and

$$
\sum_{\boldsymbol{\eta}, \eta_{6}} R\left(\boldsymbol{\eta}, \eta_{6}, B\right) \ll B \log (B)^{4} .
$$

First, we estimate the contribution of $N_{1}\left(\boldsymbol{\eta}, \eta_{6}, B\right)$. For this, we make use of the first estimate of lemma 3 to deduce that for any fixed $0<\delta \leq 1$, we have

$$
\begin{aligned}
N_{1}\left(\boldsymbol{\eta}, \eta_{6}, B\right)= & \frac{A_{2} Y_{7}}{\eta_{4} \eta_{5}} g_{2}\left(\frac{\eta_{6}}{Y_{6}} ; \boldsymbol{\eta}, B\right) \theta_{1}\left(\boldsymbol{\eta}, \eta_{6}\right) \frac{\varphi^{\circ}\left(\eta_{1}\right)}{\varphi^{\circ}\left(\operatorname{gcd}\left(\eta_{1}, \eta_{2} \eta_{4}\right)\right)} \Psi\left(\eta_{1}, \eta_{2} \eta_{3} \eta_{4} \eta_{5} \eta_{6}\right) \\
& +O\left(\frac{A_{2}}{\eta_{4} \eta_{5}} Y_{7}^{\delta} \sigma_{-\delta}\left(\eta_{1} \eta_{2} \eta_{3} \eta_{4} \eta_{5} \eta_{6}\right) \sup _{t_{7} Y_{7} \geq 1} g_{1}\left(t_{7}, \frac{\eta_{6}}{Y_{6}}\right)\right) .
\end{aligned}
$$

To estimate the overall contribution of this error term, we use the bound of lemma 6 for $g_{1}$ and we choose $\delta=1 / 4$. The average order of $\sigma_{-1 / 4}$ is $O(1)$ so we see that this contribution is

$$
\begin{aligned}
\sum_{\boldsymbol{\eta}, \eta_{6}} \sigma_{-1 / 4}\left(\eta_{1} \eta_{2} \eta_{3} \eta_{4} \eta_{5} \eta_{6}\right) \frac{A_{2} Y_{6}^{1 / 2} Y_{7}^{3 / 4}}{\eta_{4} \eta_{5} \eta_{6}^{1 / 2}} & \ll \sum_{\boldsymbol{\eta}} \sigma_{-1 / 4}\left(\eta_{1} \eta_{2} \eta_{3} \eta_{4} \eta_{5}\right) \frac{A_{2} Y_{6} Y_{7}^{3 / 4}}{\eta_{4} \eta_{5}} \\
& \ll \sum_{\eta_{1}, \eta_{2}, \eta_{3}, \eta_{5}} \sigma_{-1 / 4}\left(\eta_{1} \eta_{2} \eta_{3} \eta_{5}\right) \frac{B}{\boldsymbol{\eta}^{(1,1,1,0,1)}} \\
& \ll B \log (B)^{4},
\end{aligned}
$$


where we have summed over $\eta_{6}$ and $\eta_{4}$ using respectively the conditions (3.6) and $Y_{7} \geq 1$. Concerning the main term, we have

$$
\Psi\left(\eta_{1}, \eta_{2} \eta_{3} \eta_{4} \eta_{5} \eta_{6}\right)=\varphi^{*}\left(\eta_{2} \eta_{3} \eta_{4} \eta_{5} \eta_{6}\right) \frac{\varphi^{b}\left(\eta_{1}\right)}{\varphi^{b}\left(\operatorname{gcd}\left(\eta_{1}, \eta_{2} \eta_{4}\right)\right)}
$$

and since $\left(\eta_{1}, \eta_{2}, \eta_{4}\right) \in \mathcal{N}$, we also have

$$
\frac{\varphi^{\circ}\left(\eta_{1}\right)}{\varphi^{\circ}\left(\operatorname{gcd}\left(\eta_{1}, \eta_{2} \eta_{4}\right)\right)} \frac{\varphi^{b}\left(\eta_{1}\right)}{\varphi^{b}\left(\operatorname{gcd}\left(\eta_{1}, \eta_{2} \eta_{4}\right)\right)}=\frac{\varphi^{*}\left(\eta_{1}\right)}{\varphi^{*}\left(\operatorname{gcd}\left(\eta_{1}, \eta_{2} \eta_{4}\right)\right)} .
$$

These equalities and a short calculation prove that

$$
\theta_{1}\left(\boldsymbol{\eta}, \eta_{6}\right) \frac{\varphi^{\circ}\left(\eta_{1}\right)}{\varphi^{\circ}\left(\operatorname{gcd}\left(\eta_{1}, \eta_{2} \eta_{4}\right)\right)} \Psi\left(\eta_{1}, \eta_{2} \eta_{3} \eta_{4} \eta_{5} \eta_{6}\right)
$$

can be rewritten as $\theta_{1}^{\prime}(\boldsymbol{\eta}) \theta_{2}^{\prime}\left(\boldsymbol{\eta}, \eta_{6}\right)$ for

$$
\begin{aligned}
\theta_{1}^{\prime}(\boldsymbol{\eta}) & =\varphi^{*}\left(\eta_{1} \eta_{2} \eta_{3} \eta_{4} \eta_{5}\right) \varphi^{*}\left(\eta_{2} \eta_{3} \eta_{4} \eta_{5}\right) \frac{\varphi^{*}\left(\eta_{1} \eta_{2}\right)}{\varphi^{*}\left(\eta_{2} \eta_{4}\right)} \frac{\varphi^{*}\left(\operatorname{gcd}\left(\eta_{3}, \eta_{5}\right)\right)}{\varphi^{*}\left(\eta_{5}\right)}, \\
\theta_{2}^{\prime}\left(\boldsymbol{\eta}, \eta_{6}\right) & =\frac{\varphi^{*}\left(\eta_{6}\right)}{\varphi^{*}\left(\operatorname{gcd}\left(\eta_{6}, \eta_{3}\right)\right)} \prod_{p \mid \eta_{3}, p \nmid \eta_{2} \eta_{5} \eta_{6}}\left(1-\frac{1}{p-1}\right) .
\end{aligned}
$$

We now turn to the estimation of $N_{2}\left(\boldsymbol{\eta}, \eta_{6}, B\right)$. We only need to sum on the even $\eta_{7}$ and so, given the coprimality condition (5.4), $\eta_{2} \eta_{3} \eta_{4} \eta_{5} \eta_{6}$ is odd and thus we can make use of the first estimate of lemma 4 . The error term is the same as the previous one and, in the main term, there are exactly two differences with the case of $N_{1}\left(\boldsymbol{\eta}, \eta_{6}, B\right)$. The first is the factor $1 / 2$ and the second is the fact that here, since $\left(\eta_{1}, \eta_{2}, \eta_{4}\right) \notin \mathcal{N}$,

$$
\frac{\varphi^{\circ}\left(\eta_{1}\right)}{\varphi^{\circ}\left(\operatorname{gcd}\left(\eta_{1}, \eta_{2} \eta_{4}\right)\right)} \frac{\varphi^{b}\left(\eta_{1}\right)}{\varphi^{b}\left(\operatorname{gcd}\left(\eta_{1}, \eta_{2} \eta_{4}\right)\right)}=2 \frac{\varphi^{*}\left(\eta_{1}\right)}{\varphi^{*}\left(\operatorname{gcd}\left(\eta_{1}, \eta_{2} \eta_{4}\right)\right)}
$$

and thus we find exactly the same main term, which completes the proof of lemma 8

5.3. Summation over $\eta_{6}$. - We now proceed to sum over $\eta_{6}$. We set

$$
\mathcal{M}=\left\{\left(\eta_{3}, \eta_{2}, \eta_{5}\right) \in \mathbb{Z}_{>0}^{3}, 2 \nmid \eta_{3} \text { or } 2 \mid \eta_{2} \eta_{5}\right\} .
$$

As for the summation over $\eta_{7}$, it is clear that if $\left(\eta_{3}, \eta_{2}, \eta_{5}\right) \in \mathcal{M}$ or $2 \mid \eta_{6}$ then

$$
\prod_{p \mid \eta_{3}, p \nmid \eta_{2} \eta_{5} \eta_{6}}\left(1-\frac{1}{p-1}\right)=\prod_{\substack{p \mid \eta_{3}, p \nmid \eta_{2} \eta_{5} \eta_{6} \\ p \neq 2}}\left(1-\frac{1}{p-1}\right),
$$

and this product is equal to 0 otherwise. Furthermore, since $\eta_{2} \eta_{5}$ and $\eta_{6}$ are coprime, we have

$$
\prod_{\substack{p \mid \eta_{3}, p \nmid \eta_{2} \eta_{5} \eta_{6} \\ p \neq 2}}\left(1-\frac{1}{p-1}\right)=\frac{\varphi^{\circ}\left(\eta_{3}\right)}{\varphi^{\circ}\left(\operatorname{gcd}\left(\eta_{3}, \eta_{2} \eta_{5}\right)\right) \varphi^{\circ}\left(\operatorname{gcd}\left(\eta_{3}, \eta_{6}\right)\right)}
$$

We need to treat two cases separately depending on whether $\left(\eta_{3}, \eta_{2}, \eta_{5}\right) \in \mathcal{M}$ or $\left(\eta_{3}, \eta_{2}, \eta_{5}\right) \notin \mathcal{M}$ (note that, in the latter case, the main term of $N\left(\boldsymbol{\eta}, \eta_{6}, B\right)$ vanishes if $\left.2 \nmid \eta_{6}\right)$. Let $\mathbf{N}(\boldsymbol{\eta}, B)$ be the sum of the main term of $N\left(\boldsymbol{\eta}, \eta_{6}, B\right)$ over $\eta_{6}, \eta_{6}$ satisfying the height condition (3.6) and the coprimality condition (5.5) and let also $\mathbf{N}_{1}(\boldsymbol{\eta}, B)$ and $\mathbf{N}_{2}(\boldsymbol{\eta}, B)$ be the sums over $\eta_{6}$ respectively for $\left(\eta_{3}, \eta_{2}, \eta_{5}\right) \in \mathcal{M}$ and $\left(\eta_{3}, \eta_{2}, \eta_{5}\right) \notin \mathcal{M}$. 
Lemma 9. - We have the estimate

$$
\mathbf{N}(\boldsymbol{\eta}, B)=\zeta(2)^{-1} \frac{B}{\boldsymbol{\eta}^{(1,1,1,1,1)}} g_{3}(\boldsymbol{\eta}, B) \Theta(\boldsymbol{\eta})+\mathbf{R}(\boldsymbol{\eta}, B),
$$

where

$$
\Theta(\boldsymbol{\eta})=\frac{\varphi^{*}\left(\eta_{1} \eta_{2} \eta_{3} \eta_{4} \eta_{5}\right)}{\varphi^{\dagger}\left(\eta_{1} \eta_{2} \eta_{3} \eta_{4} \eta_{5}\right)} \varphi^{*}\left(\eta_{2} \eta_{3} \eta_{4} \eta_{5}\right) \varphi^{*}\left(\eta_{1} \eta_{2} \eta_{4} \eta_{5}\right) \frac{\varphi^{*}\left(\eta_{1} \eta_{2}\right)}{\varphi^{*}\left(\eta_{2} \eta_{4}\right)} \frac{\varphi^{*}\left(\eta_{2} \eta_{3}\right)}{\varphi^{*}\left(\eta_{2} \eta_{5}\right)}
$$

and

$$
\sum_{\boldsymbol{\eta}} \mathbf{R}(\boldsymbol{\eta}, B) \ll B \log (B)^{4} .
$$

We first treat the contribution of $\mathbf{N}_{1}(\boldsymbol{\eta}, B)$. For this, we make use of the second estimate of lemma 3 to deduce that for any fixed $0<\delta \leq 1$, we have

$$
\begin{aligned}
\mathbf{N}_{1}(\boldsymbol{\eta}, B)= & \frac{A_{2} Y_{7} Y_{6}}{\eta_{4} \eta_{5}} g_{3}(\boldsymbol{\eta}, B) \theta_{1}^{\prime}(\boldsymbol{\eta}) \frac{\varphi^{\circ}\left(\eta_{3}\right)}{\varphi^{\circ}\left(\operatorname{gcd}\left(\eta_{3}, \eta_{2} \eta_{5}\right)\right)} \Psi^{\prime}\left(\eta_{3}, \eta_{1} \eta_{2} \eta_{4} \eta_{5}\right) \\
& +O\left(\frac{A_{2} Y_{7}}{\eta_{4} \eta_{5}} Y_{6}^{\delta} \sigma_{-\delta}\left(\eta_{1} \eta_{2} \eta_{4} \eta_{5}\right) \sup _{t_{6} Y_{6} \geq 1} g_{2}\left(t_{6} ; \boldsymbol{\eta}, B\right)\right) .
\end{aligned}
$$

To estimate the overall contribution of the error term, we use the bound of lemma 6 for $g_{2}$ and we choose $\delta=1 / 4$. Since the average order of $\sigma_{-1 / 4}$ is $O(1)$, we obtain that this contribution is

$$
\begin{aligned}
\sum_{\boldsymbol{\eta}} \sigma_{-1 / 4}\left(\eta_{1} \eta_{2} \eta_{4} \eta_{5}\right) \frac{A_{2} Y_{7} Y_{6}^{3 / 4}}{\eta_{4} \eta_{5}} & \ll \sum_{\eta_{1}, \eta_{2}, \eta_{3}, \eta_{4}} \sigma_{-1 / 4}\left(\eta_{1} \eta_{2} \eta_{4}\right) \frac{B}{\boldsymbol{\eta}^{(1,1,1,1,0)}} \\
& \ll B \log (B)^{4},
\end{aligned}
$$

where we have summed over $\eta_{5}$ using the condition $Y_{6} \geq 1$. Let us turn to the main term. First, note that

$$
\frac{A_{2} Y_{7} Y_{6}}{\eta_{4} \eta_{5}}=\frac{B}{\boldsymbol{\eta}^{(1,1,1,1,1)}}
$$

In addition, we have

$$
\Psi^{\prime}\left(\eta_{3}, \eta_{1} \eta_{2} \eta_{4} \eta_{5}\right)=\varphi^{*}\left(\eta_{1} \eta_{2} \eta_{4} \eta_{5}\right) \frac{\varphi^{b}\left(\eta_{3}\right)}{\varphi^{b}\left(\operatorname{gcd}\left(\eta_{3}, \eta_{2} \eta_{5}\right)\right)} \frac{\zeta(2)^{-1}}{\varphi^{\dagger}\left(\eta_{1} \eta_{2} \eta_{3} \eta_{4} \eta_{5}\right)},
$$

and since $\left(\eta_{3}, \eta_{2}, \eta_{5}\right) \in \mathcal{M}$, we also have

$$
\frac{\varphi^{\circ}\left(\eta_{3}\right)}{\varphi^{\circ}\left(\operatorname{gcd}\left(\eta_{3}, \eta_{2} \eta_{5}\right)\right)} \frac{\varphi^{b}\left(\eta_{3}\right)}{\varphi^{b}\left(\operatorname{gcd}\left(\eta_{3}, \eta_{2} \eta_{5}\right)\right)}=\frac{\varphi^{*}\left(\eta_{3}\right)}{\varphi^{*}\left(\operatorname{gcd}\left(\eta_{3}, \eta_{2} \eta_{5}\right)\right)}
$$

An easy calculation finally yields

$$
\theta_{1}^{\prime}(\boldsymbol{\eta}) \frac{\varphi^{\circ}\left(\eta_{3}\right)}{\varphi^{\circ}\left(\operatorname{gcd}\left(\eta_{3}, \eta_{2} \eta_{5}\right)\right)} \Psi^{\prime}\left(\eta_{3}, \eta_{1} \eta_{2} \eta_{4} \eta_{5}\right)=\zeta(2)^{-1} \Theta(\boldsymbol{\eta})
$$

We now deal with the estimation of $\mathbf{N}_{2}(\boldsymbol{\eta}, B)$. We only need to sum on the even $\eta_{6}$ and so, given the coprimality condition (5.5), $\eta_{1} \eta_{2} \eta_{4} \eta_{5}$ is odd and moreover since $\left(\eta_{3}, \eta_{2}, \eta_{5}\right) \notin \mathcal{M}$, we have $2 \mid \eta_{3}$ and thus we can make use of the second estimate of lemma 4. The error term is the same as the previous one and, in the main term, there are exactly two differences with the case of $\mathbf{N}_{1}(\boldsymbol{\eta}, B)$. The first is the factor $1 / 2$ and the second is that here, since $\left(\eta_{3}, \eta_{2}, \eta_{5}\right) \notin \mathcal{N}$,

$$
\frac{\varphi^{\circ}\left(\eta_{3}\right)}{\varphi^{\circ}\left(\operatorname{gcd}\left(\eta_{3}, \eta_{2} \eta_{5}\right)\right)} \frac{\varphi^{b}\left(\eta_{3}\right)}{\varphi^{b}\left(\operatorname{gcd}\left(\eta_{3}, \eta_{2} \eta_{5}\right)\right)}=2 \frac{\varphi^{*}\left(\eta_{3}\right)}{\varphi^{*}\left(\operatorname{gcd}\left(\eta_{3}, \eta_{2} \eta_{5}\right)\right)}
$$

and we finally obtain the same main term, which concludes the proof of lemma 9 . 
5.4. Conclusion. - The aim of the following lemma is to replace the conditions $t_{6} Y_{6} \geq 1$ and $t_{7} Y_{7} \geq 1$ in the integral (5.8) defining $g_{3}$ in the main term of $\mathbf{N}(\boldsymbol{\eta}, B)$ in lemma 9 respectively by $t_{6}>0$ and $t_{7}>0$. For short, we introduce the notation

$$
D_{h}=\left\{\left(u_{2}, t_{7}, t_{6}\right) \in \mathbb{R}^{3}, t_{6}, t_{7}>0, h\left(u_{2}, t_{7}, t_{6}\right) \leq 1\right\} .
$$

Lemma 10. - For $Z_{6}, Z_{7}>0$, we have

$$
\begin{aligned}
& \operatorname{meas}\left\{\left(u_{2}, t_{7}, t_{6}\right) \in D_{h}, t_{6} Z_{6}<1\right\} \ll Z_{6}^{-1 / 2}, \\
& \operatorname{meas}\left\{\left(u_{2}, t_{7}, t_{6}\right) \in D_{h}, t_{7} Z_{7}<1\right\} \ll Z_{7}^{-1 / 2} .
\end{aligned}
$$

Proof. - These two bounds follow from the bound of lemma 6 for $g_{1}$ and the fact that $h\left(u_{2}, t_{7}, t_{6}\right) \leq 1$ implies $t_{6}, t_{7} \leq 1$.

Making use of the bound (5.13), we see that replacing the condition $t_{6} Y_{6} \geq 1$ in the integral defining $g_{3}$ in the main term of $\mathbf{N}(\boldsymbol{\eta}, B)$ in lemma 9 by the condition $t_{6}>0$ creates an error term whose overall contribution is

$$
\begin{aligned}
\sum_{\eta} \frac{A_{2} Y_{7} Y_{6}^{1 / 2}}{\eta_{4} \eta_{5}} & \ll \sum_{\eta_{1}, \eta_{2}, \eta_{3}, \eta_{4}} \frac{B}{\boldsymbol{\eta}^{(1,1,1,1,0)}} \\
& \ll B \log (B)^{4},
\end{aligned}
$$

where we have summed over $\eta_{5}$ using the condition $Y_{6} \geq 1$. The bound (5.14) shows that the same conclusion holds for the condition $t_{7} Y_{7} \geq 1$. Recalling the equality (4.2), we finally see that we can replace $g_{3}(\boldsymbol{\eta}, B)$ in the main term of $\mathbf{N}(\boldsymbol{\eta}, B)$ in lemma 9 by

$$
\iiint_{t_{6}, t_{7}>0, h\left(u_{2}, t_{7}, t_{6}\right) \leq 1} \mathrm{~d} u_{2} \mathrm{~d} t_{7} \mathrm{~d} t_{6}=\frac{\omega_{\infty}}{4} .
$$

Redefine $\Theta$ as being equal to zero if the remaining coprimality conditions (5.6) and (5.7) are not satisfied. Using lemma 5, we obtain the following result.

Lemma 11. - We have the estimate

$$
N_{U, H}(B)=\zeta(2)^{-1} \frac{\omega_{\infty}}{2} B \sum_{\boldsymbol{\eta} \in \mathcal{V}} \frac{\Theta(\boldsymbol{\eta})}{\boldsymbol{\eta}^{(1,1,1,1,1)}}+O\left(B \log (B)^{4}\right),
$$

where $\mathcal{V}$ is defined in (5.10).

Let us introduce the generalized Möbius function $\boldsymbol{\mu}$ defined for $\left(n_{1}, \ldots, n_{5}\right) \in \mathbb{Z}_{>0}^{5}$ by $\boldsymbol{\mu}\left(n_{1}, \ldots, n_{5}\right)=\mu\left(n_{1}\right) \cdots \mu\left(n_{5}\right)$. We set $\mathbf{k}=\left(k_{1}, k_{2}, k_{3}, k_{4}, k_{5}\right)$ and we define, for $s \in \mathbb{C}$ such that $\Re(s)>1$,

$$
\begin{aligned}
F(s) & =\sum_{\boldsymbol{\eta} \in \mathbb{Z}_{>0}^{5}} \frac{|(\Theta * \boldsymbol{\mu})(\boldsymbol{\eta})|}{\eta_{1}^{s} \eta_{2}^{s} \eta_{3}^{s} \eta_{4}^{s} \eta_{5}^{s}} \\
& =\prod_{p}\left(\sum_{\mathbf{k} \in \mathbb{Z}_{\geq 0}^{5}} \frac{\left|(\Theta * \boldsymbol{\mu})\left(p^{k_{1}}, p^{k_{2}}, p^{k_{3}}, p^{k_{4}}, p^{k_{5}}\right)\right|}{p^{k_{1} s} p^{k_{2} s} p^{k_{3} s} p^{k_{4} s} p^{k_{5}}}\right) .
\end{aligned}
$$

It is easy to see that if $\mathbf{k} \notin\{0,1\}^{5}$ then $(\Theta * \boldsymbol{\mu})\left(p^{k_{1}}, p^{k_{2}}, p^{k_{3}}, p^{k_{4}}, p^{k_{5}}\right)=0$ and moreover if exactly one of the $k_{i}$ is equal to 1 , then $(\Theta * \boldsymbol{\mu})\left(p^{k_{1}}, p^{k_{2}}, p^{k_{3}}, p^{k_{4}}, p^{k_{5}}\right) \ll 1 / p$, so the local factors $F_{p}$ of $F$ satisfy

$$
F_{p}(s)=1+O\left(\frac{1}{p^{\min (\Re(s)+1,2 \Re(s))}}\right) .
$$


This proves that $F$ actually converges in the half-plane $\Re(s)>1 / 2$, which implies that $\Theta$ satifies the assumption of [LB10, Lemma 8]. Applying this lemma, we get

$$
\sum_{\boldsymbol{\eta} \in \mathcal{V}} \frac{\Theta(\boldsymbol{\eta})}{\boldsymbol{\eta}^{(1,1,1,1,1)}}=\alpha\left(\sum_{\boldsymbol{\eta} \in \mathbb{Z}_{>0}^{5}} \frac{(\Theta * \boldsymbol{\mu})(\boldsymbol{\eta})}{\boldsymbol{\eta}^{(1,1,1,1,1)}}\right) \log (B)^{5}+O\left(\log (B)^{4}\right),
$$

where $\alpha$ is the volume of the polytope defined in $\mathbb{R}^{5}$ by $t_{1}, t_{2}, t_{3}, t_{4}, t_{5} \geq 0$ and

$$
\begin{aligned}
& t_{1}+2 t_{2}+3 t_{3}+2 t_{5} \leq 1, \\
& 3 t_{1}+2 t_{2}+t_{3}+2 t_{4} \leq 1 .
\end{aligned}
$$

A computation using Franz's additional Maple package [Fra09] provides $\alpha=1 / 2160$, that is to say

$$
\alpha=2 \alpha(\widetilde{V}) .
$$

Moreover,

$$
\begin{aligned}
\sum_{\boldsymbol{\eta} \in \mathbb{Z}_{>0}^{5}} \frac{(\Theta * \boldsymbol{\mu})(\boldsymbol{\eta})}{\boldsymbol{\eta}^{(1,1,1,1,1)}} & =\prod_{p}\left(\sum_{\mathbf{k} \in \mathbb{Z}_{\geq 0}^{5}} \frac{(\Theta * \boldsymbol{\mu})\left(p^{k_{1}}, p^{k_{2}}, p^{k_{3}}, p^{k_{4}}, p^{k_{5}}\right)}{p^{k_{1}} p^{k_{2}} p^{k_{3}} p^{k_{4}} p^{k_{5}}}\right) \\
& =\prod_{p}\left(1-\frac{1}{p}\right)^{5}\left(\sum_{\mathbf{k} \in \mathbb{Z}_{\geq 0}^{5}} \frac{\Theta\left(p^{k_{1}}, p^{k_{2}}, p^{k_{3}}, p^{k_{4}}, p^{k_{5}}\right)}{p^{k_{1}} p^{k_{2}} p^{k_{3}} p^{k_{4}} p^{k_{5}}}\right) .
\end{aligned}
$$

The remaining coprimality conditions greatly simplify the calculation and we obtain

$$
\sum_{\mathbf{k} \in \mathbb{Z}_{\geq 0}^{5}} \frac{\Theta\left(p^{k_{1}}, p^{k_{2}}, p^{k_{3}}, p^{k_{4}}, p^{k_{5}}\right)}{p^{k_{1}} p^{k_{2}} p^{k_{3}} p^{k_{4}} p^{k_{5}}}=\left(1-\frac{1}{p^{2}}\right)^{-1}\left(1-\frac{1}{p}\right)\left(1+\frac{6}{p}+\frac{1}{p^{2}}\right),
$$

which gives

$$
\sum_{\boldsymbol{\eta} \in \mathbb{Z}_{>0}^{5}} \frac{(\Theta * \boldsymbol{\mu})(\boldsymbol{\eta})}{\boldsymbol{\eta}^{(1,1,1,1,1)}}=\zeta(2) \prod_{p}\left(1-\frac{1}{p}\right)^{6} \omega_{p}
$$

We complete the proof of theorem 1 putting together the equalities (5.15), (5.16), (5.17) and lemma 11.

\section{References}

[BB07] R. de la Bretèche and T. D. Browning. On Manin's conjecture for singular del Pezzo surfaces of degree 4. I. Michigan Math. J., 55(1):51-80, 2007.

[BD09] T. D. Browning and U. Derenthal. Manin's conjecture for a quartic del Pezzo surface with $A_{4}$ singularity. Ann. Inst. Fourier (Grenoble), 59(3):1231-1265, 2009.

[BT98] V. V. Batyrev and Y. Tschinkel. Manin's conjecture for toric varieties. J. Algebraic Geom., 7(1):15-53, 1998.

[CLT02] A. Chambert-Loir and Y. Tschinkel. On the distribution of points of bounded height on equivariant compactifications of vector groups. Invent. Math., 148(2):421-452, 2002.

[CT88] D. F. Coray and M. A. Tsfasman. Arithmetic on singular Del Pezzo surfaces. Proc. London Math. Soc. (3), 57(1):25-87, 1988.

[Der06a] U. Derenthal. Geometry of universal torsors. PhD thesis, Georg-August-Universität Göttingen, 2006.

[Der06b] U. Derenthal. Singular del Pezzo surfaces whose universal torsors are hypersurfaces. arXiv:math/0604194v1, 2006. 
[Der07] U. Derenthal. On a constant arising in Manin's conjecture for del Pezzo surfaces. Math. Res. Lett., 14(3):481-489, 2007.

[Der09] U. Derenthal. Counting integral points on universal torsors. Int. Math. Res. Not. IMRN, (14):2648-2699, 2009.

[DL10] U. Derenthal and D. Loughran. Singular del Pezzo surfaces that are equivariant compactifications. Zapiski Nauchnykh Seminarov (POMI), 377:26-43, 2010.

[DT07] U. Derenthal and Y. Tschinkel. Universal torsors over del Pezzo surfaces and rational points. In Equidistribution in number theory, an introduction, volume 237 of NATO Sci. Ser. II Math. Phys. Chem., pages 169-196. Springer, Dordrecht, 2007.

[FMT89] J. Franke, Y. I. Manin, and Y. Tschinkel. Rational points of bounded height on Fano varieties. Invent. Math., 95(2):421-435, 1989.

[Fra09] M. Franz. Convex - a Maple package for convex geometry, version 1.1, 2009.

[LB10] P. Le Boudec. Manin's conjecture for two quartic del Pezzo surfaces with $3 \mathbf{A}_{1}$ and $\mathbf{A}_{1}+\mathbf{A}_{2}$ singularity types. Acta Arith., to appear, arXiv:1006.0691v2, 2010.

[Lou10] D. Loughran. Manin's conjecture for a singular sextic del Pezzo surface. J. Théor. Nombres Bordeaux, 22(3):675-701, 2010.

[Pey95] E. Peyre. Hauteurs et mesures de Tamagawa sur les variétés de Fano. Duke Math. J., 79(1):101-218, 1995.

Pierre Le Boudec, Université Denis Diderot (Paris VII), Institut de Mathématiques de Jussieu, UMR 7586, Case 7012 - Bâtiment Chevaleret, Bureau 7C14, 75205 Paris Cedex 13, France E-mail : pleboude@math.jussieu.fr 